\title{
A novel direction finding algorithm using ICA with uniform circular array
}

\author{
Li Wanchun \\ dept. Electronic Engineering \\ University of Electronic Science and Technology of China \\ Chengdu, China \\ liwanchun@uestc.edu.cn
}

\author{
Li Yingxiang \\ dept. Communication Engineering \\ Chengdu University of Information Technology \\ Chengdu, China
}

\begin{abstract}
In this paper, a novel algorithm for twodimensional angle estimation with uniform circular array (UCA) based on independent component analysis (ICA) is proposed. First the linear mixed signals are separated by ICA. Then, separated signals are used to waveform matching filter with the original mixed-signal. Next, The array manifold is obtained with permutation and scaling problems, using the first rows to divide every rows, and using angle algorithm to the modify manifold matrix, obtain the angle information by the least mean square algorithm, thus pair-marching for elevation and azimuth automatically. Finally, Monte Carlo simulations show the effectiveness of the proposed method.
\end{abstract}

Keywords-Direction Finding, Independent Component Analysis, Uniform Circular Array

\section{INTRODUCTION}

Direction finding (DF) using UCA (uniform circular array) has been discussed in many literatures 1-7, there are many methods to solve this problem, such as multiple signal characterization (MUSIC), ESPRIT based on MODE and so on As ESPRIT method uses the approximation of Bessel function at moderate number arrays, it can not work at less arrays; MUSIC method uses the orthonormality of the signal's subspace and the noise's subspace, thus it needs moderate arrays. In literature1, a closed form solution for DF is proposed by using the symmetry of UCA, but it can only work in case of one target. A new DOA algorithm based on ICA is proposed in this paper, which can be used in multi-target, and pairmarching for elevation and azimuth. Assume that the signals are uncorrelated, separating the signals by ICA from the mix of arrays, using the separated signals and the received array signals to find the array manifold, and the azimuth angles and elevation angle can be obtained by least square method.

This paper is organized as follows. In section 2, the model for uniform circular array signal is given. In section 3 , the proposed DF algorithm based on ICA is presented. The perturbation analysis is presented in section 4. Computer experimental results of the algorithm proposed in this paper is also presented in section 5. Finally, our work of this paper is summarized in the last section.

\section{MODEL FOR UNIFORM CIRCULAR ARRAY SIGNAL}

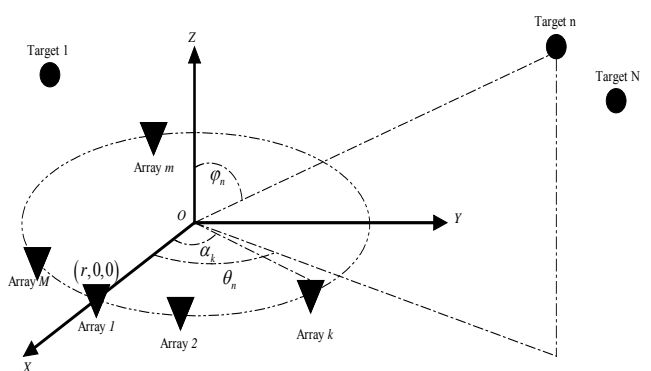

Fig.1 Uniform Circular array Configuration

The received signal can be written by:

$$
\begin{gathered}
X_{k}(t)=\sum_{n=1}^{N} e^{j \frac{2 \pi r}{\lambda} \cos \left(\theta_{n}-\alpha_{k}\right) \sin \left(\varphi_{n}\right)} s_{n}(t)+v_{k}(t) \\
t=1,2, \cdots, T, \quad k=1,2, \cdots, M \quad, \quad n=1,2, \cdots, N
\end{gathered}
$$

Where: $T$ denotes the snapshots, $M$ is the number of arrays, $N$ is the number of source.

Assume that the received signal is ergodic in time domain, the noise is white in space-time, that is:

$$
\left\{\begin{array}{c}
E\left\{v_{k}(t) v_{m}{ }^{*}(\tau)\right\}=\sigma_{v}{ }^{2} \delta(k-m) \delta(t-\tau) \\
E\left\{v_{k}(t) v_{m}(\tau)\right\}=0
\end{array}\right.
$$

The signals have the property:

$$
\left\{\begin{array}{c}
E\left\{s_{k}(t) s_{m}{ }^{*}(\tau)\right\}=\sigma_{s k}{ }^{2} \delta(k-m) \delta(t-\tau) \\
E\left\{s_{k}(t) s_{m}(\tau)\right\}=0
\end{array}\right.
$$

Equation (1) can be written in matrix form:

$$
\mathbf{x}(t) \boldsymbol{\varphi s} \mathbf{A}(, \mathbf{v})(t)+(t)
$$

Where:

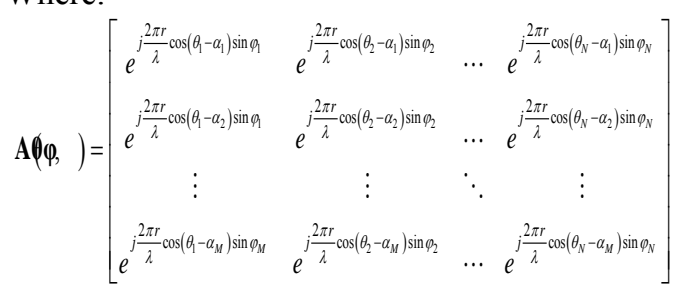




$$
\begin{aligned}
\mathbf{s}(t) & =\left[\begin{array}{lll}
s_{1}(t) & \cdots & s_{N}(t)
\end{array}\right]^{T} ; \\
\mathbf{v}(t) & =\left[\begin{array}{lll}
v_{1}(t) & \cdots & v_{M}(t)
\end{array}\right]^{T} ; \\
\mathbf{x}(t) & =\left[\begin{array}{lll}
x_{1}(t) & \cdots & x_{M}(t)
\end{array}\right]^{T}
\end{aligned}
$$

\section{METHOD OF DIRECTION FINDING}

Firstly, the number of source can be estimated by AIC, $\mathrm{MDL}^{[8]}$ or $\mathrm{GDE}^{[9-10]}$. Thus, we can assume the number of source $M$ is known.

As the signals which are received by the UCA are complex signals, we can only use complex ICA algorithms. Taking into account the convergence of the ICA, Iterative ICA algorithm is to use, for the common signal, which includes circular signals and noncircular signals, so the $\mathrm{NC}_{-}$fastICA ${ }^{[11]}$ algorithm is used to separate the received signals.

The estimated signals $\hat{\mathbf{s}}(t)$ and $\mathbf{W}$ can be obtained by

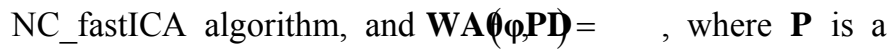
permutation matrix, $\mathbf{D}$ is a diagonal matrix. Matching Filter algorithm is used with the received signals $\mathbf{x}(t)$ and the estimated signals $\hat{\mathbf{s}}(t)$. White the other signals, and reserve one signal, and use the DOA algorithm for one angle, the DOA is obtained and pair-marching for elevation and azimuth.

Let:

$$
\mathbf{Y}=E\left[\mathbf{x}(t) \hat{\mathbf{s}}^{H}(t)\right]=\mathbf{A}(\theta, \varphi) \mathbf{P D}+\mathbf{V}
$$

where: $\mathbf{v}$ follows the gauss distribution,

$\mathbf{v} \sim C N\left(\mathbf{0}, \sigma^{2} \mathbf{P}_{\hat{\mathbf{s}}}\right), \quad \mathbf{P}_{\hat{\mathbf{s}}}=E\left(\mathbf{s}(t) \hat{\mathbf{s}}^{H}(t)\right)$

In the case of high SNR, the noise can be ignored, so:

$$
\mathbf{Y}=\mathbf{A}(\theta, \varphi) \mathbf{P D}
$$

In the ideal condition for ICA algorithm, noted that:

$$
\mathbf{P D}=\left[\begin{array}{cccc}
0 & 0 & & 0 \\
0 & 0 & & d_{N} \\
\vdots & d_{2} & & 0 \\
d_{1} & \vdots & \ddots & \vdots \\
\vdots & 0 & & 0 \\
0 & 0 & & 0
\end{array}\right]
$$

That is: only one nonzero number in every column for matrix PD. Assume that the nonzero number $\lambda_{p}$ in $m_{p}$ row for the $p$-th column.

Taking equation (7) into equation (4), yields:

$$
\begin{gathered}
\mathbf{Y}=\left[\begin{array}{cccc}
d_{m_{1}} e^{j \frac{2 \pi r}{\lambda} \cos \left(\theta_{m_{1}}-\alpha_{1}\right) \sin \varphi_{m_{1}}} & d_{m_{2}} e^{j \frac{2 \pi r}{\lambda} \cos \left(\theta_{m_{2}}-\alpha_{1}\right) \sin \varphi_{m_{2}}} & \cdots & d_{m_{N}} e^{j \frac{2 \pi r}{\lambda} \cos \left(\theta_{m_{N}}-\alpha_{1}\right) \sin \varphi_{m_{N}}} \\
d_{m_{1}} e^{j \frac{2 \pi r}{\lambda} \cos \left(\theta_{m_{1}}-\alpha_{2}\right) \sin \varphi_{m_{1}}} & d_{m_{2}} e^{j \frac{2 \pi r}{\lambda} \cos \left(\theta_{m_{2}}-\alpha_{2}\right) \sin \varphi_{m_{2}}} & \cdots & d_{m_{N}} e^{j \frac{j \pi r}{\lambda} \cos \left(\theta_{m_{N}}-\alpha_{2}\right) \sin \varphi_{m_{N}}} \\
\vdots & \vdots & \ddots & \vdots \\
d_{m_{1}} e^{j \frac{2 \pi r}{\lambda} \cos \left(\theta_{m_{1}}-\alpha_{M}\right) \sin \varphi_{m_{1}}} & d_{m_{2}} e^{j \frac{2 \pi r}{\lambda} \cos \left(\theta_{m_{2}}-\alpha_{M}\right) \sin \varphi_{m_{2}}} & \cdots & d_{m_{N}} e^{j \frac{2 \pi r}{\lambda} \cos \left(\theta_{m_{N}}-\alpha_{M}\right) \sin \varphi_{m_{N}}}
\end{array}\right] \\
\mathbf{Y} \triangleq\left[\begin{array}{cccc}
y_{11} & y_{12} & \cdots & y_{1 N} \\
y_{21} & y_{22} & \cdots & y_{2 N} \\
\vdots & \vdots & \ddots & \vdots \\
y_{M 1} & y_{M 2} & \cdots & y_{M N}
\end{array}\right]
\end{gathered}
$$

Noted that there is one unknown coefficient $d_{m_{p}}$, in order to eliminate the influence, every rows is divided by the first row, According to the $m_{p}$ row:

$$
\left[\begin{array}{c}
e^{j \frac{2 \pi r}{\lambda_{p}}\left[\cos \left(\theta_{m_{p}}-\alpha_{2}\right) \sin \varphi_{m_{p}}-\cos \left(\theta_{m_{p}}-\alpha_{1}\right) \sin \varphi_{m_{p}}\right]} \\
\vdots \\
\left.e^{j \frac{2 \pi r}{\lambda_{p}}\left[\cos \left(\theta_{m_{p}}-\alpha_{M}\right) \sin \varphi_{m_{p}}-\cos \left(\theta_{m_{p}}-\alpha_{1}\right) \sin \varphi_{m_{p}}\right.}\right]
\end{array}\right]=\left[\begin{array}{c}
\frac{y_{2 m_{p}}}{y_{1 m_{p}}} \\
\vdots \\
\frac{y_{M m_{p}}}{y_{1 m_{p}}}
\end{array}\right]
$$

Get the angle to the both side of equation (10), assume that no phase ambiguity in there:

$$
\begin{aligned}
& {\left[\begin{array}{c}
\sin \varphi_{m_{p}} \cos \theta_{m_{p}}\left(\cos \alpha_{2}-\cos \alpha_{1}\right)-\sin \varphi_{m_{p}} \sin \theta_{m_{p}}\left(\sin \alpha_{2}-\sin \alpha_{1}\right) \\
\vdots \\
\sin \varphi_{m_{p}} \cos \theta_{m_{p}}\left(\cos \alpha_{M}-\cos \alpha_{1}\right)-\sin \varphi_{m_{p}} \sin \theta_{m_{p}}\left(\sin \alpha_{M}-\sin \alpha_{1}\right)
\end{array}\right]} \\
& =\frac{\lambda_{p}}{2 \pi r}\left[\begin{array}{c}
\operatorname{angle}\left(y_{2 m_{p}} / y_{1 m_{p}}\right) \\
\vdots \\
\text { angle }\left(y_{M m_{p}} / y_{1 m_{p}}\right)
\end{array}\right]
\end{aligned}
$$

Note that:

$$
\begin{aligned}
& \cos \left(\theta_{m_{p}}-\alpha_{2}\right) \sin \varphi_{m_{p}}-\cos \left(\theta_{m_{p}}-\alpha_{1}\right) \sin \varphi_{m_{p}} \\
& =\left[\cos \left(\theta_{m_{p}}-\alpha_{2}\right)-\cos \left(\theta_{m_{p}}-\alpha_{1}\right)\right] \sin \varphi_{m_{p}} \in[-2,2] \\
& \text { Because angle }\left(y_{2 m_{p}} / y_{1 m_{p}}\right) \in[-\pi, \pi], \text { so if } 2(2 \pi r) \leq \pi \lambda, \text { that }
\end{aligned}
$$
is $r \leq \lambda / 4$, there is no phase ambiguity in equation (11). On the other hand, the smaller of $r$, the smaller effective aperture of the UCA, so we suggest that in this algorithm, the $r$ is selected as $\lambda / 4$.

Let:

$$
\begin{aligned}
& \mathbf{z} \boldsymbol{\varsigma}=\left[\begin{array}{c}
\sin \varphi_{m_{p}} \cos \theta_{m_{p}} \\
\sin \varphi_{m_{p}} \sin \theta_{m_{p}}
\end{array}\right] ; \quad=\frac{\lambda_{p}}{2 \pi r}\left[\begin{array}{c}
\operatorname{angle}\left(y_{2 m_{p}} / y_{1 m_{p}}\right) \\
\vdots \\
\operatorname{angle}\left(y_{M m_{p}} / y_{1 m_{p}}\right)
\end{array}\right] \\
& \mathbf{F}=\left[\begin{array}{cc}
\cos \alpha_{2}-\cos \alpha_{1} & \sin \alpha_{2}-\sin \alpha_{1} \\
\vdots & \vdots \\
\cos \alpha_{M}-\cos \alpha_{1} & \sin \alpha_{M}-\sin \alpha_{1}
\end{array}\right]
\end{aligned}
$$

We have:

$$
\mathbf{F z}=
$$

That is:

$$
\mathbf{z}=\left(\mathbf{F}^{H} \mathbf{F}\right)^{-1} \mathbf{F}^{H}
$$

So we get:

$$
\theta_{m_{p}}=\arctan \frac{\mathbf{z}(2)}{\mathbf{z}(1)}, \varphi_{m_{p}}=\arcsin \sqrt{\mathbf{z}^{T} \mathbf{z}}
$$

\section{PERTURBATION ANALYSIS}

In fact, in equation 8 , the column of matrix PD does not include only one non-zero element, for the incompleteness of ICA method, there are others non-zero elements in the column 
of matrix PD, Without loss of generality, we analyze the first column elements of matrix PD, assume that the major element is $d_{1}$ and lie in the first row, the mini element is $\varepsilon_{1}$ and lie in the second row, and $\left|\varepsilon_{1}\right|<<\left|d_{1}\right|$, as there are noise in the receive datum, so every element of matrix PD is noisy, based on equation (2) and (3), the noise from ICA on the elements of each row is also a zero-mean Gaussian white noise, assume that wavelength is $\lambda$, the first column elements of matrix PD can be written as :

$$
\mathbf{y}=\left[\begin{array}{c}
d_{1} e^{j \frac{2 \pi r}{\lambda} \cos \left(\theta_{1}-\alpha_{1}\right) \sin \varphi_{1}}+\varepsilon_{1} e^{j \frac{2 \pi r}{\lambda} \cos \left(\theta_{2}-\alpha_{1}\right) \sin \varphi_{2}}+u_{1} \\
d_{1} e^{j \frac{2 \pi r}{\lambda} \cos \left(\theta_{1}-\alpha_{2}\right) \sin \varphi_{1}}+\varepsilon_{1} e^{j \frac{2 \pi r}{\lambda} \cos \left(\theta_{2}-\alpha_{2}\right) \sin \varphi_{2}}+u_{2} \\
\vdots \\
d_{1} e^{j \frac{2 \pi r}{\lambda} \cos \left(\theta_{1}-\alpha_{M}\right) \sin \varphi_{1}}+\varepsilon_{1} e^{j \frac{2 \pi r}{\lambda_{2}} \cos \left(\theta_{2}-\alpha_{M}\right) \sin \varphi_{2}}+u_{M}
\end{array}\right]
$$

So the equation (10) becomes:

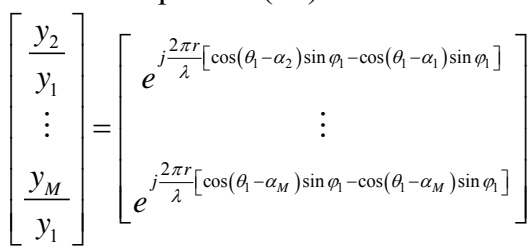

$$
+\left[\begin{array}{c}
\frac{\varepsilon_{1}\left(e^{j \frac{2 \pi r}{\lambda} \cos \left(\theta_{2}-\alpha_{1}\right) \sin \varphi_{2}}-e^{j \frac{2 \pi r}{\lambda} \cos \left(\theta_{2}-\alpha_{2}\right) \sin \varphi_{2}}\right)+u_{2}-u_{1}}{d_{1} e^{j \frac{2 \pi r}{\lambda} \cos \left(\theta_{1}-\alpha_{1}\right) \sin \varphi_{1}}+\varepsilon_{1} e^{j \frac{2 \pi r}{\lambda} \cos \left(\theta_{2}-\alpha_{1}\right) \sin \varphi_{2}}+u_{1}} \\
\vdots \\
\frac{\varepsilon_{1}\left(e^{j \frac{2 \pi r}{\lambda} \cos \left(\theta_{2}-\alpha_{M}\right) \sin \varphi_{2}}-e^{j \frac{2 \pi r}{\lambda} \cos \left(\theta_{2}-\alpha_{2}\right) \sin \varphi_{2}}\right)+u_{M}-u_{1}}{d_{1} e^{j \frac{2 \pi r}{\lambda} \cos \left(\theta_{1}-\alpha_{1}\right) \sin \varphi_{1}}+\varepsilon_{1} e^{j \frac{2 \pi r}{\lambda} \cos \left(\theta_{2}-\alpha_{1}\right) \sin \varphi_{2}}+u_{1}}
\end{array}\right]
$$

Taking first order Taylor Series Expansion, we get:

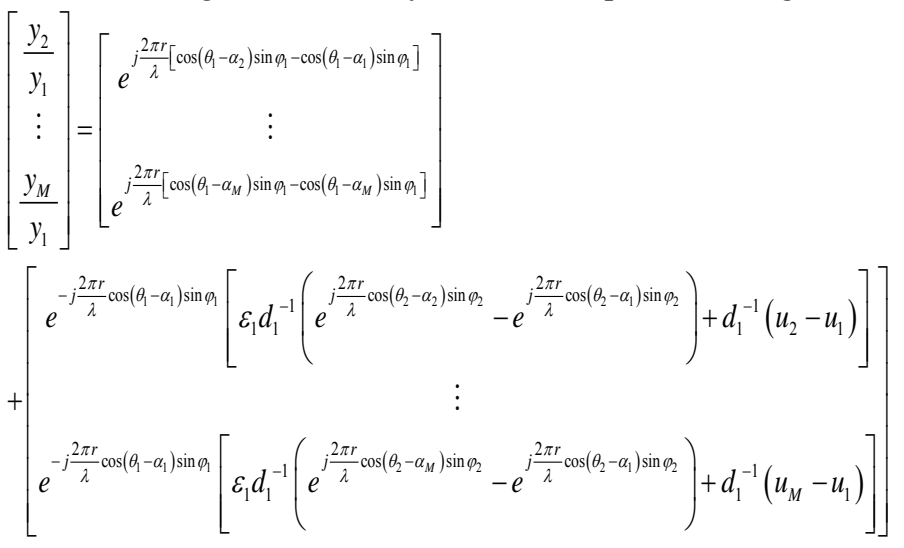

In order to simplify operations, let:

$$
\begin{aligned}
& \beta_{k}=\frac{2 \pi r}{\lambda}\left[\cos \left(\theta_{k}-\alpha_{2}\right) \sin \varphi_{1}-\cos \left(\theta_{k}-\alpha_{1}\right) \sin \varphi_{1}\right] \\
& \delta_{r k}+j \delta_{j k}=e^{-j \frac{2 \pi r}{\lambda} \cos \left(\theta_{1}-\alpha_{1}\right) \sin \varphi_{1}} \\
& *\left[\varepsilon_{1} d_{1}^{-1}\left(e^{j \frac{2 \pi r}{\lambda} \cos \left(\theta_{2}-\alpha_{k}\right) \sin \varphi_{2}}-e^{j \frac{2 \pi r}{\lambda} \cos \left(\theta_{2}-\alpha_{1}\right) \sin \varphi_{2}}\right)+d_{1}^{-1}\left(u_{k}-u_{1}\right)\right]
\end{aligned}
$$

Equation (19) can be written as:

$$
\left[\begin{array}{c}
y_{2} / y_{1} \\
\vdots \\
y_{M} / y_{1}
\end{array}\right]=\left[\begin{array}{c}
e^{j \beta_{2}}+\delta_{r 2}+j \delta_{j 2} \\
\vdots \\
e^{j \beta_{M}}+\delta_{r M}+j \delta_{j M}
\end{array}\right]
$$

In the case of high SNR, $\left|u_{k}\right|<<\left|d_{k}\right|,\left|\varepsilon_{k}\right|<<\left|d_{k}\right|$, we get: $\left|\delta_{r k}\right|=1,\left|\delta_{j k}\right|=1$. Taking angle information in the equation (21), and using first order Taylor Series Expansion, we yields:

$$
\text { angle }\left(\left[\begin{array}{c}
y_{2} / y_{1} \\
\vdots \\
y_{M} / y_{1}
\end{array}\right]\right)=\left[\begin{array}{c}
\beta_{2} \\
\vdots \\
\beta_{M}
\end{array}\right]+\left[\begin{array}{c}
\cos \beta_{2} \delta_{j 2}-\sin \beta_{2} \delta_{r 2} \\
\vdots \\
\cos \beta_{M} \delta_{j M}-\sin \beta_{M} \delta_{r M}
\end{array}\right]
$$

So the perturbation of equation (15) is given by:

$$
\tilde{\mathbf{z}}=\left(\mathbf{F}^{H} \mathbf{F}\right)^{-1} \mathbf{F}^{H \sim}
$$

where

$$
\tilde{\boldsymbol{\varsigma}}=\left[\begin{array}{c}
\cos \beta_{2} \delta_{j 2}-\sin \beta_{2} \delta_{r 2} \\
\vdots \\
\cos \beta_{M} \delta_{j M}-\sin \beta_{M} \delta_{r M}
\end{array}\right]
$$

\section{EXPERIMENTAL RESULTS}

Computer simulations have been carried out to evaluate the performance of the proposed method for 2$\mathrm{D}$ direction finding of narrowband sources in the presence of additive white Gaussian noise. We consider UCA consisting of varying antennas. There are three independent sinusoidal signal, their DOAs are $\left(20^{\circ}, 20^{\circ}\right)$, $\left(40^{\circ}, 50^{\circ}\right),\left(80^{\circ}, 70^{\circ}\right)$. The number of samples is 1000 . We employ the joint distribution estimation of azimuth and elevation angle and the mean square error (MSE) as the performance measure and all results provided are averages of 10000 independent runs. At the condition that UCA consisting of three antennas, and the radius $r=\lambda / 4$. Fig. 2 shows the joint distribution estimation of azimuth and elevation angle, the Fig. 2 (a) is at $\mathrm{SNR}=20 \mathrm{~dB}$ and (b) is at $\mathrm{SNR}=30 \mathrm{~dB}$, Figs. 3 shows the MSEs with the three source's azimuth and elevation angle, (a) shows the azimuth and (b) shows the elevation, respectively.

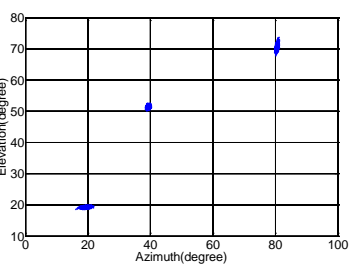

(a)

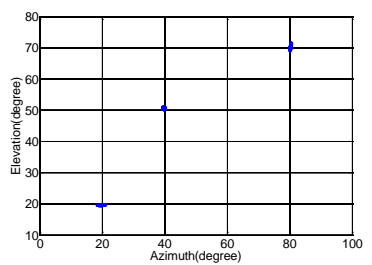

(b)
Fig.2 The Joint distribution estimation of azimuth and elevation angle 


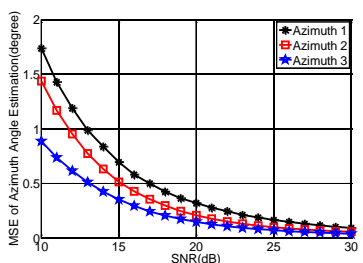

(a)

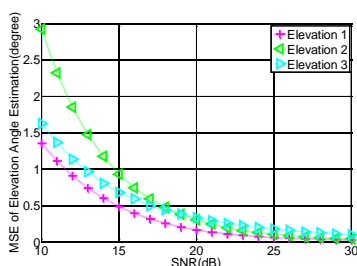

(b)
Fig3. MSE of azimuth angle and elevation angle

At the condition that UCA consisting of four antennas, and the radius $r=\lambda / 4$. Fig. 4 shows the joint distribution estimation of azimuth and elevation angle, the Fig. 4(a) is at $\mathrm{SNR}=20 \mathrm{~dB}$ and (b) is at $\mathrm{SNR}=30 \mathrm{~dB}$, Figs. 5 shows the MSEs with the three source's azimuth and elevation angle, (a) shows the azimuth and (b) shows the elevation, respectively.

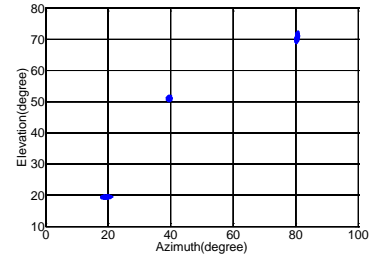

(a)

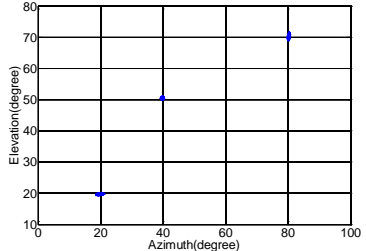

(b)
Fig.4 The Joint distribution estimation of azimuth and elevation angle

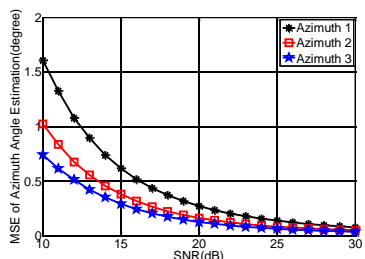

(a)

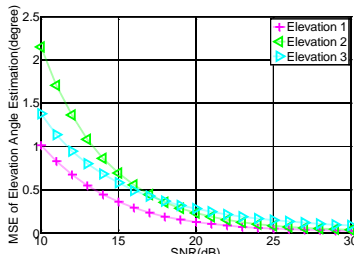

(b)
Fig5. MSE of azimuth angle and elevation angle

At the condition that UCA consisting of five antennas, and the radius $r=\lambda / 4$. Fig. 6 shows the joint distribution estimation of azimuth and elevation angle, the Figs.6 (a) is at $\mathrm{SNR}=20 \mathrm{~dB}$ and $(\mathrm{b})$ is at $\mathrm{SNR}=30 \mathrm{~dB}$, Figs. 7 shows the MSEs with the three source's azimuth and elevation angle, (a) shows the azimuth and (b) shows the elevation, respectively. (a)

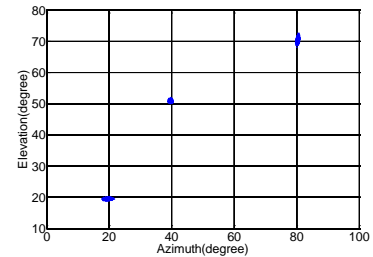

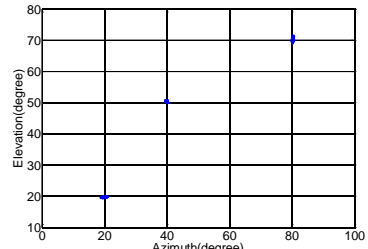

(b)
Fig.6 The Joint distribution estimation of azimuth and elevation angle

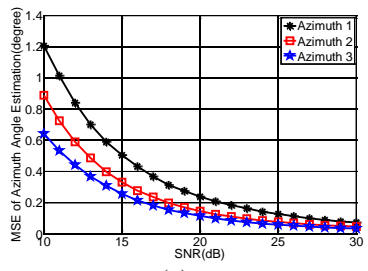

(a)

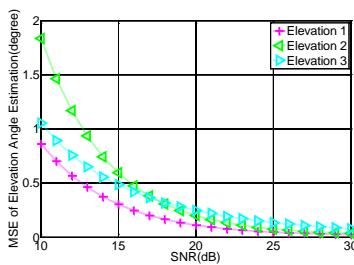

(b)
Fig7. MSE of azimuth angle and elevation angle

From the simulations, the proposed method can bring to well performance for multi-signals direction finding at moderate SNR, pair-marching for elevation and azimuth can be automatic, and can obtain precision DOA estimation with less arrays.

\section{CONCLUSIONS}

A novel algorithm for 2-D angle estimation of narrowband sources with a UCA has been devised. The key idea in the algorithm development is to utilize the ICA to obtain separated signals, and using them to get the array manifold. It is shown that the proposed algorithm has a well estimation performance at sufficiently high SNR conditions.

\section{ACKNOWLEDGMENT}

This work was supported by Meteorological information and Signal Processing Key Laboratory of Sichuan Higher Education Institutes.

\section{REFERENCES}

[1] $\mathrm{Wu}, \mathrm{Y}$. and H. C. So, "Simple and accurate two-dimensional angle estimation for a single source with uniform circular array," IEEE Antenna Wireless Propagat. Lett., Vol. 7, 78-80, 2008.

[2] R. O. Schmidt, "Multiple emitter location and signal parameter estimation," IEEE Trans. Antennas Propag., vol. AP-34, no. 3, pp. 271280, Mar. 1986.

[3] C. P. Mathews and M. D. Zoltowski, "Eigenstructure techniques for 2-D angle estimation with uniform circular arrays," IEEE Trans. Signal Process., vol. 42, no. 9, pp. 2395-2407, Sep. 1994.

[4] Z. Ye, L. Xiang, and X. Xu, "DOA estimation with circular array via spatial averaging algorithm," IEEE Antennas Wireless Propag. Lett., vol. 6, pp. 74-76, 2007.

[5] J.-J. Fuchs, "On the application of the global matched filter to DOA estimation with uniform circular arrays," IEEE Trans. Signal Process., vol. 49, no. 4, pp. 702-709, Apr. 2001.

[6] K. R. Sundaram, R. K. Mallik, and U. M. S. Murthy, "Modulo conversion method for estimating the direction of arrival," IEEE Trans. Aerosp. Electron. Syst., vol. 36, no. 4, pp. 1391-1396, 2000.

[7] Roy R, Kailath T. ESPRIT-estimation of signal parameter via rotational invairance techques. IEEE Trans.SP,Vol.37 No.7, 984-999, 1989

[8] M. Wax and T.Kailath "Detection of signals by information theoretic criteria” IEEE Trans. ASSP Vol ASSP-33 No.2 387-392 Apr.1985.

[9] Wu H T, Yang J F, chen F K. Sources Number estimator Using Gerschgorin Disks. Proc ICASSP, Adelaide, Australia, 1994, 261-264.

[10] O. Caspary, P. Nus, T. Cecchin, The source number estimation based on Gerschgorin radii, Acoustics, Speech and Signal Processing, 1998. Proceedings of the 1998 IEEE International Conference on Volume 4, 12-15 May 1998 Page(s):1993 - 1996 vol.4.

[11] M. Novey,T. Adali. On extending the complex Fast ICA algorithm to noncircular sources.IEEE Trans. Signal Processing. 2008, 56(5):21482154 